\title{
LA SOLEDAD Y LA TRISTEZA DE NATHANIEL HAWTHORNE: UNA ESPECULACIÓN BIOGRÁFICA*
}

\author{
Carlos J. Madrid Jurado**
}

\begin{abstract}
Resumen: Son muchas las leyendas surgidas en torno al carácter solitario y taciturno de Nathaniel Hawthorne. Su aversión al mundo, la muerte del padre cuando el novelista tenía sólo cuatro años, su invalidez a los nueve, su timidez y rechazo a todo contacto físico o la intensa, si bien algo fría, relación de la madre con los hijos han servido tradicionalmente de argumento para explicar la solitud de Hawthorne. Sin embargo, este artículo pretende destacar que la soledad y la tristeza del novelista estuvieron profundamente marcadas por su propia fantasía, don éste que el autor no consiguió ni entender ni aceptar completamente. Palabras clave: Literatura Norteamericana del siglo XIX, Hawthorne, biografía, tristeza, fantasía
\end{abstract}

\begin{abstract}
Many legends have evolved as a result of Nathaniel Hawthorne's solitary and melancholy nature. His aversion to the world, his father's death when the author was only four, his invalidity at the age of nine, his shyness and dislike towards physical contact or the intense but somewhat cold bond of the mother to her children are some of the major points which have traditionally been used to account for Hawthorne's solitude. In this article, however, I endeavour to highlight that the novelist's isolation and melancholy were greatly influenced by his own fancy, a gift which he neither understood nor accepted completely.
\end{abstract}

Keywords: 19th-century American Literature, Hawthorne, biography, sadness, fancy

Si para Charles F. Hoffman hay algo que realmente distingue los cuentos y sketches de Twice Told Tales en comparación con la obra de otros escritores es el tono de aflicción que persiste en todos ellos (1998: 70). Para entender la profunda e inevitable tristeza de Nathaniel Hawthorne y que Luedtke atribuye a la influencia que tuvo sobre su ficción la cultura oriental (1989: 93), debemos remontarnos a la niñez del autor y en concreto a dos sucesos de trascendental importancia para él. Por un lado, la repentina muerte del padre, el capitán Hathorne ${ }^{1}$, en el transcurso de uno de sus largos viajes por el mar cuando el escritor

\footnotetext{
Fecha de recepción: febrero 2006

Fecha de aceptación y versión final: septiembre 2006

** Profesor I.E.S. Andrés de Vandelvira, Baeza (Jaén) y profesor tutor de la UNED; $\triangle$ cjmadrid04@yahoo.es.

${ }^{1}$ Se ha especulado mucho sobre el momento y las razones que llevaron a Hawthorne a añadir una "w" al apellido paterno. Sampson justifica el hecho diciendo: "[...] after leaving college [Hawthorne] changed the spelling of the name, finding in some of his researches into family history that it had been spelled with the " $w$ " in England, and realizing that the pronunciation was becoming vitiated to match the spelling" (1964: 298).
}

Odisea, $n^{\circ} 7$, ISSN $1578-3820,2006,119-126$ 
aún no había cumplido los cuatro años de edad. Hawthorne nunca se refirió al suceso expresamente, pero fue tal la huella que dejó en él que la figura paterna sería prácticamente inexistente en toda su obra. A partir de aquel fatídico día la vida familiar, según recogen los informes de familiares y biógrafos, cambió por completo. La madre se recluía en su habitación para prácticamente no salir de ella, ni siquiera para comer, quedando así toda la casa sumida en lo que Chandler califica de "a serious and quiet atmosphere" (2002: 5). Pero eso no sería todo. Los Mannings, parientes de los Hathorne por parte materna, pasaron a hacerse cargo del bienestar de la viuda y de sus tres hijos, un gesto de generosidad que, según parece, no fue nunca suficiente para ganar la confianza y llegar al corazón del joven Nathaniel, que no tardaría en mostrar claros síntomas de resentida aflicción. Prueba de ello son las tristes palabras: “And I'm never coming home again” (citado en Miller 1991: 26), con las que el adolescente daba fin a sus primeros cuentos y con las que parecía no haber terminado de asumir el que su padre hubiese salido un día de casa para nunca más volver. Quizá el contundente final pudiera atribuirse también a cierto espíritu de rebeldía ante la nueva situación económica y familiar presidida por sus tíos, hacia los cuales siempre mantuvo una postura distante y ambigua. Sea como fuere, no hay duda de que el sentimiento de desvalimiento por su temprana orfandad unido a esa relación, intensa si bien algo fría, ${ }^{2}$ de la madre hacia sus hijos, fueron factores determinantes en la carrera del novelista y los ingredientes de uno de sus primeros y más bellos cuentos: "The Gentle Boy". El relato es toda una recreación sincera de la lúgubre niñez del autor, marcada por la tristeza y el abandono, y que ocho años antes de morir rememoraría en una de sus cartas a su amigo Horatio Bridge a propósito de "[...] the gloom and chill of [his] early life" (Letters, XVII: 497).

El otro suceso que resultó decisivo en la vida del novelista, no sólo porque lo encaminaría hacia el mundo de las letras, sino porque contribuyó a aumentar aún más si cabe su carácter reservado y melancólico, fue el accidente que sufrió en el pie siendo niño y que lo obligó a llevar muletas desde los nueve hasta los doce años. Durante ese tiempo, Hawthorne se vio forzado a abandonar los juegos propios de un chico de su edad para convertirse en un ávido lector, mostrándose cada vez más distante de los demás. Este aislamiento se convirtió en algo habitual años después durante su estancia en la pequeña ciudad de Raymond (Maine). Su editor, James T. Fields, recordaba en relación a todo esto que Hawthorne, en cierta ocasión, le había contado en una de sus cartas que, si bien el tiempo que pasó en dicho lugar fue para él el más feliz de su vida, fue también allí donde empezó a condenarse a la soledad (1979: 139).

Al mencionar la tristeza del novelista, sale a relucir su soledad, algo que no es extraño si tenemos en cuenta que en muchas ocasiones una acompaña inevitablemente a la otra. No obstante, y en el caso particular de Hawthorne, hemos de decir que la imagen que tradicionalmente se ha tenido de él como la de un hombre melancólico y tímido, no es una simple deducción lógica de esa tristeza inicial que comentábamos. Entre sus libros de

\footnotetext{
${ }^{2}$ Dos días antes de morir su madre, Hawthorne confesaba esa separación que siempre hubo entre ellos dos: "I love my mother" -decía- "but there has been, ever since my boyhood, a sort of coldness of intercourse between us, such as is apt to come between persons of strong feelings, if they are not managed rightly" (American Notebooks, 429).
}

Odisea, $\mathrm{n}^{\circ}$ 7, ISSN 1578-3820, 2006, 119-126 
notas, prefacios, cartas y cuentos se encuentran numerosos comentarios que aluden a esa reclusión que nunca ocultó. De todos ellos resulta especialmente significativo aquél en el que el estadounidense relacionaba su aversión a la sociedad con la naturaleza melancólica de su carácter. Según el narrador de "Fragments from the Journal of a Solitary Man"3, lo que verdaderamente distinguía a Oberon -seudónimo que Hawthorne empezó a utilizar en su época de estudiante en Bowdoin- era "a sadness [...] on his spirit", ingrediente éste que añadido a "the shrinking sensitiveness of his nature, rendered him not misanthropic, but singularly averse to social intercourse" (XII, 24).

De todas las alusiones hechas por biógrafos, críticos y estudiosos acerca de la soledad de Hawthorne destaca, por la trascendencia que tuvo sobre el novelista y su obra, aquélla que da cuenta de los doce años que pasó recluido, leyendo y escribiendo, en el ático del domicilio materno, con la esperanza de llegar algún día a ser conocido por el gran público. En relación a este periodo son famosas las palabras que el escritor dirigió en 1837 en una de sus cartas a su amigo Longfellow. "It gratifies me" -le comentaba-, "to find that you have occasionally felt an interest in my situation; but your quotation [...] about the 'larknest,' makes me laugh", para seguidamente añadir:

You would have been much nearer to the truth, if you had pictured me as a dwelling in an owl's nest; for mine is about as dismal, and, like the owl, I seldom venture abroad till after dusk. By some witchcraft or other-I have been carried apart from the main current of life, and find it impossible to get back again. Since we last met [ . . . ] I have secluded myself from society; and yet I never meant any such thing, nor dreamed what sort of life I was going to lead. I have made a captive of myself and put me in a dungeon; and now I cannot find the key to let myself out -and if the door were open, I should be almost afraid to come out. (Letters, $\mathrm{XV}: 251)$

Es imposible saber si Hawthorne, conociendo de antemano los efectos negativos que a la postre le traería tal encierro, habría optado voluntariamente por la misma vía, u otra diferente, para alcanzar su sueño. Posiblemente nunca reparó en ello. Su ambición a la sazón por llegar a ser un gran escritor era demasiado fuerte como para hacerle ver de manera clara los pros y contras de una soledad que, por otro lado, le había acompañado durante tanto tiempo que nada hacía sospechar que pudiera finalmente rebelarse contra él de la forma que luego lo hizo. Cuando en 1835, después de llevar diez años enclaustrado y a sólo dos de entablar su contacto con el mundo, éste le confesaba a sus lectores lo que luego habría de decirle a Longfellow, su separación del mundo había llegado a tal extremo que ésta ya no le permitía volver atrás, no porque a lo largo de ese tiempo Hawthorne hubiese perdido completamente la capacidad de decidir libremente, sino porque, como bien explicaba Darrel a propósito de la Ley del Karma ${ }^{4}$ " "the effect of such process [was] to limit the possibilities of choice" (1953: 377).

\footnotetext{
${ }^{3}$ Todas las notas y citas en relación a los cuentos y sketches mencionados en este artículo han sido tomadas de la edición The Complete Works of Nathaniel Hawthorne with Introductory Notes by George Parsons Lathrop. Boston: Houghton Mifflin and Company, The River Side Presss. 1883. 13 vols.

${ }^{4}$ Esta ley budista sostiene la creencia de que toda causa (palabra, pensamiento o acción) produce un efecto que no sabemos cuándo, cómo ni dónde va a tener lugar.
} 
Conociendo pues las nefastas consecuencias que para el ser humano tiene toda decisión de aislarse de los demás, no extraña que Hawthorne procurara convencer al lector para que no siguiera sus mismos pasos. Así, en "The Prophetic Pictures", el novelista no dudaría en alertarnos de que, en efecto, "it is not good for a man to cherish a solitary ambition unless there be those around him by whose example he may regulate himself, his thoughts, desires, and hopes will become extravagant" (I, 207), advertencia que tendría ocasión de repetir un año más tarde, concretamente en su cuento "Peter Goldthwaite's Treasure", cuando insta a todo individuo a "[rectify] his mind by other minds, and [square] in conduct to that of his neighbors, so as seldom to be lost in eccentricity" (I, 447). Buenos consejos sin duda, pero nada válidos para un hombre, ya en ese tiempo, enormemente sofisticado, como él mismo manifestó, cuando, a propósito de esa tendencia suya a la soledad, confesaba una incapacidad patológica para sentir el calor de los demás al mismo tiempo que para seguir sus propios consejos (Letters, XVI: 325).

Fuera lo que fuere, no parece que la causa de ese aislamiento estuviese en la relación del novelista con su mujer, hijos y amigos, pues su vida, aunque con avatares, fue, comparándola con la de otros escritores contemporáneos, relativamente tranquila y afortunada, no así su mundo interior, el cual, al parecer, veíase a menudo abrumado por fantasmas que le impedían armonizar la parte social, profesional y privada de su vida de la forma que a él le hubiese gustado. Waggoner sostiene al hilo de este desequilibrio emocional que a Hawthorne siempre le resultó difícil "to feel as he knew he ought to feel" (1952: 4). Y es que junto al Hawthorne cordial que algunos biógrafos han ensalzado siempre coexistió otro bien distinto; un hombre que, salvando los primeros años de su matrimonio, nunca consiguió ser feliz, mostrándose constantemente insatisfecho con su propio trabajo, y en ocasiones incluso contrariado por lo que su propio genio le incitaba a escribir. Esa permanente incapacidad suya para actuar y sentir a cada momento de la manera más adecuada de acuerdo a las circunstancias no pudo por menos que afectar de igual manera a su obra literaria. El comentario de Hawthorne a su editor, Fields, confesándole no entender el sentido real que había querido dar a aquellas "blasted allegories" de Mosses from an Old Manse (Letters, XVII, 201) apunta a que, en el momento de escribir, algún elemento extraño hacía que sus relatos tomaran un camino diferente al deseado para convertirse al final en algo que no sólo repudiaba sino que le hacía repudiarse a sí mismo con igual intensidad, tal y como podemos ver en su misma carta a propósito de la citada colección. Desde aquellos tiempos de Mosses, escribía Hawthorne, "I am a good deal changed [...]; and to tell you the truth, my past self is not very much to my taste, as I see myself in this book [...]. It has been a very disagreeable task to read the book" (Letters, XVII, 201). Curiosamente, ese sentimiento de angustia por no poder controlar ni entender lo que en determinados momentos su fantasía le dictaba ya había aparecido impreso casi veinte años antes en uno de sus cuadernos de notas, prueba de que aquella sensación de pérdida de control que hiciera llegar a Fields desde Liverpool en abril de 1854 ya le había torturado mucho tiempo antes. He aquí el revelador apunte: "A person to be writing a tale, and to find that it shapes against his intentions; that the characters act otherwise than he thought; that unforeseen events occur; and a catastrophe comes which he strives in vain to alert. It might shadow forth his own fate, -he having made himself one of the personages (IX, 28).

Odisea, $\mathrm{n}^{\circ}$ 7, ISSN 1578-3820, 2006, 119-126 
Esa, digamos, doble y a su vez misteriosa personalidad del novelista, fue incluso reconocida por quienes mejor lo conocieron. Su amigo Jonathan Cilley diría: "I love Hawthorne, I admire him, but" -matizaba- "I do not know him. He lives in a mysterious world of thought and imagination which he never permits me to enter" (en Miller, 1991: 74). Su propia esposa, Sophia, confesaba no ser capaz de mirarle fijamente a los ojos a menos que éstos permanecieran cerrados para de esta forma no profanar lo que ella describía como "a holy place" (9). Hasta el último momento -reconocía ésta, como ya hiciera antes el yerno de ambos, George Parson Lathrop-, "he was in a measure to me a divine Mystery, for he was so to himself" (9), dando con ello a entender, no ya lo poco que llegó a conocer a su marido, sino el desconocimiento que éste tenía de sí mismo, especialmente de ese lado artístico suyo, sublime y turbulento, que yacía bajo ese aspecto suyo de hombre sosegado.

Más reveladoras, aún si cabe, son las declaraciones hechas en esta misma línea por el hijo del escritor, Julian Hawthorne, y un amigo de aquél, George Hillard, en torno al contraste que, según ellos, había entre la persona del novelista y su obra. Éste último, recordemos, se preguntaba todo perplejo de dónde podía venirle al genio, no ya esa afición por la mórbida anatomía del corazón humano, sino su conocimiento al respecto. $\mathrm{Al}$ leer sus cuentos -comentaba-, uno toma la impresión de que su autor se encuentra abrumado por alguna pena oculta; de que, en algún lugar de su alma existe una lúgubre morada a la que ni él mismo se atreve a entrar (en Miller, 1991: 8). Por su parte, Julian, después de haber leído los cuentos y las novelas de su progenitor, decía sentirse incapaz de comprender cómo un hombre como su padre había podido escribir unos libros así. En su opinión terminaba diciendo-, hombre y escritor eran, en lo que a Hawthorne se refiere, tan diferentes como la noche y el día (J. Hawthorne I, 1884: 96).

"The artist -the true artist- must look beneath the exterior" (I, 202), decía Hawthorne en los primeros años de su carrera apostando por la fantasía y alertando del riesgo inminente que corre el hombre de ser engañado por esa falsa verdad que el mundo exterior trata de imponerle como única y auténtica. Contra ese intento de imposición, sólo la imaginación puede llevar al escritor a contemplar el alma de las cosas -la verdadera realidad-, haciéndole superar toda barrera procedente del mundo que le rodea. "To see the inmost soul" (I, 202), es sin duda alguna el don que define por antonomasia a los hijos predilectos del arte y el que distinguió, por encima de cualquier otra cualidad, a Hawthorne, quien, no obstante, al hablar de ese "his gift" (I, 202), no hizo por ocultar la agridulce complacencia que tal facultad le producía, calificándola como "his proudest but often a melancholy one" (I, 202). Esa brillante a la vez que melancólica imaginación se deja entrever por doquier en todos los relatos que escribió a lo largo de su carrera literaria. Diríase que es un elemento ineludible de su ficción que aparece aquí y allá de la forma más imprevisible, incluso en aquellos sketches de tema más desenfadado, como son las meditaciones del autor propiciadas por una sugerente e intrascendente tormenta de nieve en "Snowflakes", o el paseo de éste de la mano de una niña en "Little Annie's Ramble", y en los que las ensoñaciones del narrador, aunque alegres en un principio, terminan siendo -en palabras de Toulouse-, "personally disquieting" (1991:208).

Este sentimiento de desasosiego que solía con frecuencia apoderarse del alma del escritor en la mayoría de sus cuentos no parece que se debiera, según decíamos, a otra cosa 
que a la aflicción que en los más profundo de su ser le producía la prodigiosa habilidad con que su imaginación conseguía traspasar los muros de las sórdidas celdas de la condición humana -especialmente aquéllos "which communicate with the infernal regions" (II, 197)-, una prerrogativa que sólo poseen los adeptos del diablo, tal como deja explícito el propio Hawthorne en aquella escena de "Young Goodman Brown" donde Satanás se dirige en el oscuro corazón del bosque a cuantos se han congregado en su nombre:

By the sympathy of your human hearts for sin ye shall scent out all the places -whether in church, bedchamber, street, field, or forest — where crime has been committed [...]. Far more than this. It shall be yours to penetrate, in every bosom, the deep mystery of sin, the fountain of all wicked arts, and which inexhaustibly supplies more evil impulses than human powerthan my power at its utmost- can make manifest in deeds. (II, 103-4)

Hawthorne, cierto es, nunca gozó de ese conocimiento diabólico que distingue a los hijos de Satanás. Sobre la posibilidad real de realizar tan perversa intrusión en el corazón del individuo, el autor dejaría dicho en uno de sus primeros relatos, allá por 1830, que, "if I would know the interior of brick walls, or the mystery of human bosoms, I can but guess" (I, 220). Fue, no obstante, la desbocada fantasía del genio, siempre rebelde en estos casos hacia los dictados y consejos de la razón, la que aparece desde un principio en su obra claramente seducida por cuanto hay de misterioso en el alma. En el siguiente fragmento vemos cómo el joven escritor sueña por unos instantes con la posibilidad de penetrar allí donde él por sí mismo jamás podría llegar. El deseo de traspasar los límites humanos es tan fuerte e irreprimible que su imaginación, inspirada por cuanto logra contemplar desde la torre de un campanario, se deja transportar por unos instantes de la mano del picaresco Diablo Cojuelo de Le Sage -éste a su vez una réplica del personaje de Luis Vélez de Guevara-, para a continuación recrearse en lo fascinante que sería adoptar como "the most desirable mode of existence" (I, 220) el espectro de Paul Pry ${ }^{5}$. He aquí las palabras del novelista:

Oh that the Limping Devil of Le Sage would perch beside me here, extend his wand over this contiguity of roofs, uncover every chamber, and make me familiar with their inhabitants! The most desirable mode of existence might be that of a spiritualized Paul Pry, hovering invisible round man and woman, witnessing their deeds, searching into their hearts, borrowing brightness from their felicity, and shade from their sorrow, and retaining no emotion peculiar to himself. (I, 220)

$\mathrm{Si}$, como parece cierto, "fancy provides the illusion of a self with creative freedom" (Toulouse 1991: 210), es importante también destacar que no todo lo que ésta concede al individuo, y en particular al escritor, resulta conveniente, ya que "fanciful associations can also lead to dangerous feelings of self-loss" (210). Esa arma de doble filo que es la imaginación no sólo dio lo mejor de sí misma a Hawthorne, sino que, pensamos, también le hizo ver

\footnotetext{
${ }^{5}$ Personaje que dio título a una obra publicada en 1825 por el dramaturgo norteamericano John Poole (1786-1872), el cual gozó de cierto renombre entre sus contemporáneos. Otra de las creaciones de este escritor, Hamlet Travestie, publicada en 1811, puso de moda las versiones burlescas de algunas de las obras de Shakespeare (Ousby 1992: 787).
}

Odisea, $\mathrm{n}^{\circ} 7$, ISSN 1578-3820, 2006, 119-126 
su lado más oscuro, el cual nunca terminó de aceptar. Si bien la idea de verse a sí mismo siempre le sedujo al escritor, tanto que entre sus preferencias se encontraba la de "to make one's own reflection in a mirror the subject of a story" (IX, 26), verdad es que todas los intentos que hizo en este sentido en su ficción no hacen sino poner de manifiesto la tortura que le propinaba el hecho de aceptarse tal cual. Basta recordar la zozobra del novelista en "Monsieur du Miroir" al mirarse introspectivamente frente al espejo, o el horror del protagonista en "The Minister's Black Veil”. En este último cuento Parson Hooper, un clérigo de una de las iglesias de Nueva Inglaterra, oculta, abrumado por un misteriosa e inconfesable pena, su rostro al mundo tras un infranqueable velo negro. El terror que se tiene a sí mismo y que aumenta con su negativa a mostrar esa parte oculta de su ser es tal que comenta el narrador: "he never willingly passed before a mirror, nor stooped to drink at a still fountain, lest, in its peaceful bosom, he should be affrighted by himself" (I, 64).

Hawthorne no sólo sintió miedo por su lado más oculto dominado por una descontrolada y desbordante fantasía. La no aceptación de esa habilidad también le provocó al estadounidense, como no podía ser de otra manera, sentimientos de culpa, sobre todo cuando aquélla se ponía en su obra al servicio del mal. En "Fancy's Show Box", Hawthorne se pregunta si acaso un escritor no es "[a] malicious artist" (I, 253) por el mero hecho de poseer el don de crear en su imaginación excéntricos malvados. Pero dejemos que sean las palabras del narrador las que hablen por sí mismas:

A scheme of guilt, till it be put in execution, greatly resembles a train of incidents in a projected tale. The latter, in order to produce a sense of reality in the reader's mind, must be conceived with such proportionate strength by the author as to seem, in the glow of fancy, more like truth, past, present, or to come, than purely fiction. The prospective sinner, on the other hand, weaves his plot of crime, but seldom or never feels a perfect certainty that it will be executed. There is a dreaminess diffused about his thoughts; in a dream, as it were, he strikes the death-blow into his victim's heart, and starts to find an indelible blood-stain on his hand. Thus a novel writer or a dramatist, in creating a villain of romance and fitting him with evil deeds, and the villain of actual life, in projecting crimes that will be perpetrated, may almost meet each other half-way between reality and fancy. (I, 256)

Aunque imaginaria, no debe resultarnos ni extraña ni exagerada esta visión inicua de uno mismo, y menos aún en el caso de Hawthorne, que no dejaba dudas a la hora de reconocer en "P.'S Correspondence" la enorme influencia que sobre sí mismo ejerce la imaginación al admitir: "it is sometimes a serious question with me whether ideas be not really visible and tangible, and endowed with all the other qualities of matter" (II, 410). No en vano fue reconocido por Henry James como "a man of Fancy" (1997: 48). Esa habilidad natural del escritor de Salem para radiografiar los abismos de la condición humana no sólo lo separó del mundo y de los suyos, sino que, para su soledad y tristeza, quedó prisionero de ella.

\section{REFERENCIAS BIBLIOGRÁFICAS}

ChandleR, E. L. 2002. A Study of the Sources of the Tales and Romances Written by Nathaniel Hawthorne before 1853. Ann Arbor, MI: Smith College Studies in Modern Languages (facsímil). 
DARRel, A. 1953. "The Devil in Boston.” Philological Quarterly 32: 361-68.

Fields, J. T. 1979. "Hawthorne”. Ed C. J. Dello Buono. Rare Early Essays on Nathaniel Hawthorne. Norwood, PA: Norwood Editions, Rare Early Essays Series: 65-151.

Hawthorne, J. 1884. Nathaniel Hawthorne and His Wife. 2 vols. Boston: Houghton Mifflin.

Hawthorne, N. 1883. The Complete Works of Nathaniel Hawthorne with Introductory Notes by George Parsons Lathrop. River Side Edition. 13 Vols. Boston: Houghton, Mifflin and Company.

1972. The American Notebooks. The Centenary Edition of the Works of Nathaniel Hawthorne. Ed. R. H. Pearce \& C. M. Simpson. Columbus, OH: Ohio State UP.

1984-87. The Letters: 1813-1843. Ed. T. Woodson, L. Neal Smith \& Norman H. Pearson. Ohio: Ohio State University Press. Vol. 15 of The Centenary Edition of the Works of Nathaniel Hawthorne. 20 vols.

. 1985-87. The Letters: 1843-1853. Eds. T. Woodson, L. Neal SmitH and Norman H. Pearson. Ohio: Ohio State University Press. Vol. 16 of The Centenary Edition of the Works of Nathaniel Hawthorne. 20 vols.

. 1985-87. The Letters: 1853-1856. Eds. T. Woodson, L. Neal Smith \& Norman Holmes Pearson. Ohio: Ohio State University Press. Vol. 17 of The Centenary Edition of the Works of Nathaniel Hawthorne. 20 vols.

JAMES, H. 1997. Hawthorne. Ithaca and London: Cornell University Press.

HolfFman, C. F. 1998. "Review" (5 March 1838). Nathaniel Hawthorne. Critical Assessments I. Ed. Brian HARDING. Robertsbridge: Helm Information, 68-70.

LuedtKe, L. S. 1989. Nathaniel Hawthorne and the Romance of the Orient. Bloomington and Indianapolis: Indiana University Press.

Miller, E. H. 1991. Salem is My Dwelling Place. A Life of Nathaniel Hawthorne. Duckworth: University of Iowa.

Ousby, I., ed. 1992. The Cambridge Guide to Literature in English. Cambridge: Cambridge University Press.

SAmpson, E. C. 1964. "The 'W' in Hawthorne's Name." Essex Institute Historical Collections 100: 297-299.

Toulouse, T. 1991. "Seeing through 'Paul Pry': Hawthorne's Early Sketches and the Problem of Audience." Critical Essays on Hawthorne's Short Stories. Ed. Albert J. VON FRANK. Boston: G. K. Hall, 203-219.

WaGgoner, H. H. 1955. Hawthorne. A Critical Study. Rev. Edition. Cambridge, MA.: Harvard University Press. 